\title{
VALORACIÓN DEL CAMBIO AMBIENTAL SEGÚN LOS "JUNQUEROS" Y "LEÑATEROS" EN EL SECTOR SUR DE LA REGIÓN RIOPLATENSE (ARGENTINA)
}

\author{
PABLO C. STAMPELLA ${ }^{1,2}$, MARÍA B. DOUMECQ ${ }^{1}$, MATILDE VOJKOVIC ${ }^{1}$ \& LUCIANA LABORDA ${ }^{1}$
}

\begin{abstract}
Summary: Stampella, P. C., M. B. Doumecq, M. Vojkovic \& L. Laborda. 2016. Assessment of environmental change according to "junqueros" and "leñateros" in the southern sector of the Río de la Plata region (Argentina). Bonplandia 25(1): 17-31.

Through an ethnoecological approach, 24 interviews to key informants were made in Magdalena and Punta Indio districts, Rio de la Plata region, Argentina. The aim was collecting and evaluating junqueros and leñateros local narratives regarding environmental change and its impact on their daily lives. The extractive activities: fuelwood and rushes collection, were very important in this area, although they have declined nowadays, especially the latter one. Two events have influenced the activity: oil spill and changes in production systems. As a result of the establishment of the "Parque Costero del Sur", a biosphere reserve, modifications in the fuelwood activity were registered. However, unlike the rushes collection, the abundance of wood and the reformulation of selection practices and strategies have made it possible to continue this activity, despite the changes in the study area. Each activity by the key informants influenced the narratives about their assessment of the environmental change, since few changes were in common among them and recognized in relation to climate and wildlife.
\end{abstract}

Key words: Extractive activities, local narratives, Magdalena, Punta Indio, Rio de la Plata coast.

Resumen: Stampella, P. C., M. B. Doumecq, M. Vojkovic \& L. Laborda. 2016. Valoración del cambio ambiental según los "junqueros" y "leñateros" en el sector sur de la región rioplatense (Argentina). Bonplandia 25(1): 17-31.

Mediante un abordaje etnoecológico, se realizaron 24 entrevistas a informantes calificados de los partidos de Magdalena y Punta Indio, en el litoral rioplatense, con el objetivo de recopilar y evaluar las narrativas de junqueros y leñateros locales respecto del cambio ambiental y su impacto en sus vidas cotidianas. Estas actividades extractivas (obtención de leña y recolección de juncos) fueron muy importantes en esta zona, aunque en la actualidad sufren una merma, sobre todo en la actividad junquera, debido principalmente a dos acontecimientos que marcaron el rumbo de la actividad: derrame de petróleo y cambios en los sistemas productivos. En la actividad leñera se registraron cambios principalmente como producto de la creación de la Reserva de Biosfera "Parque Costero del Sur", sin embargo, a diferencia de los junqueros, la abundancia del recurso leñatero y la reformulación en las prácticas y estrategias de selección hizo posible la permanencia de la actividad a pesar de los cambios sufridos en el área de estudio. Las actividades ejercidas por los informantes influyeron en las narrativas sobre su valoración del cambio ambiental, dado que se reconocieron pocos cambios en común, principalmente, en relación con el clima y la fauna.

${ }^{1}$ Laboratorio de Etnobotánica y Botánica Aplicada (LEBA), Facultad de Ciencias Naturales y Museo, Universidad Nacional de La Plata, 64 Nro.3, La Plata (1900), Argentina.

${ }^{2}$ Becario Posdoctoral CONICET. E-mail: pstampella@yahoo.com 
Palabras clave: Actividades extractivas, litoral rioplatense, Magdalena, narrativas locales, Punta Indio.

\section{Introducción}

Con frecuencia, el término "entorno" se utiliza como sinónimo de "ambiente" o "medio", en referencia al espacio físico donde se encuentran los seres vivos. Este planteo usualmente deja de lado las diversas interacciones del organismo con otros organismos, de la misma especie y de otras, que son parte de su entorno. De este modo, reducir el entorno al espacio físico circundante lleva implícita una separación (o dicotomía) entre el organismo y aquello que lo rodea. Esto se extiende a los seres humanos y su entorno (los seres humanos somos organismos), y en este contexto se hace evidente, por lo general, una segunda disociación, entre un medio "natural" y otro "cultural", dimensiones difíciles de separar (Hurrell \& Albuquerque, 2012; Hurrell, 2014) en estrecha relación de interdependencia (Maffi, 2001).

Etter et al. (2008) proponen que el "ambiente" debe ser entendido como un concepto "social", o más bien como una extensión de lo "social" en lo "natural", e integran para el abordaje de estas problemáticas a las ciencias humanas y las naturales a través de nuevos conocimientos sobre la ontogenia de la ciencia del cambio global. Emplearemos el término "ambiente" en este sentido, relacionando "naturaleza" y "cultura" de manera conjunta.

Por otra parte, el concepto de "cambio ambiental" no tiene una definición clara y precisa. Numerosos trabajos se refieren a ese concepto, sin definirlo (García Codrón \& Silió Cervera, 2000; Parlee et al., 2012; Richeri et al., 2013). En 1998, Primack y otros (Gerritsen et al., 2002), de manera muy general, definen el "cambio ambiental" como una transición gradual entre dos situaciones, cuyo resultado puede ser considerado de menor o mayor valor que el inicial. Esta definición descontextualizada, se puede referir a cualquier cambio, por ejemplo, un cambio económico o político; incluso, un cambio de ánimo. Otra definición de "cambio ambiental (global)" es la brindada por Vitousek (1992): "cambios que alteran las envolturas fluidas bien mezcladas del sistema Tierra (atmósfera y océanos) experimentados globalmente, pero también aquellos que ocurren en sitios discretos pero de amplio efecto que constituyen un cambio global". Los "globales" se refieren a los cambios climáticos, en la composición atmosférica, disminución de la concentración de ozono e incremento de los rayos UV. Los "locales" son cambios en el uso de la tierra, pérdida de biodiversidad, invasiones biológicas y cambios locales en la dinámica atmosférica.

El método más empleado para dilucidar las causas y los procesos del cambio ambiental se basa en la evaluación cuantitativa del mismo y su efecto en los componentes bióticos (Carroll et al., 2003; Manzoli et al., 2011; Valdés \& García, 2011), relegando muchas veces la dimensión histórica del proceso de cambio. No obstante, algunos trabajos consideran el enfoque diacrónico, incluyendo datos derivados del análisis de imágenes satelitales y/o de documentación histórica (Etter et al., 2008; Viedma et al., 2015; Wang et al., 2015). Sin embargo, para el abordaje de problemáticas locales es poco frecuente contar con información de este tipo, por lo cual el enfoque etnoecológico se vuelve relevante al respecto (Lane, 1997; Parlee et al., 2012; Celentano et al., 2014).

El enfoque etnoecológico implica el estudio holístico y multidisciplinario de los sistemas de conocimientos, creencias y prácticas que los diferentes grupos humanos tienen sobre su ambiente (Toledo, 1992, 2002), focalizándose en los actores sociales que participan de una red de relaciones, localizada en tiempo y espacio (Nazarea, 1999).

De esta manera, la interpretación de las narrativas y prácticas de los actores sociales acerca del cambio ambiental permite la caracterización de los cambios locales y del conocimiento ecológico local (Hurrell et al., 2015). En este marco, es imprescindible considerar las historias de vida y las particularidades de cada actor entrevistado, debido a que los recuerdos varían según sus intereses y profesiones $\mathrm{y}$, por tanto, sus 
discursos sobre el ambiente. De acuerdo con Lane (1997), las personas acumulan conocimientos a través de su propia experiencia y las de las generaciones pasadas, pero el proceso de memoria está relacionado con las experiencias de vida y es, necesariamente, muy selectivo.

La presente contribución incluye resultados preliminares de un estudio actualmente en curso en el sector sur de la región rioplatense, acerca de la valoración local del cambio ambiental. El objetivo fue registrar y evaluar las narrativas de "junqueros" (personas que, para el consenso del ámbito local y regional intervienen en la manufactura del junco, Schoenoplectus californicus (C.A. Mey.) Soják, y en especies afines (Cyperaceae), mayormente cortadores) y "leñateros" (personas que cortan, recolectan y utilizan leña), quienes realizan dos actividades típicas en el área de estudio, respecto del cambio ambiental local y/o regional y su impacto en sus vidas cotidianas. Se considera que las prácticas extractivas implican una relación directa de los actores sociales con el ambiente y los recursos naturales de los que hacen uso a diario, en este caso, el junco y la leña.

\section{Materiales y Métodos}

\section{Área de Estudio}

La región rioplatense abarca el área de influencia del río de La Plata, desde el delta inferior, su frente de avance, la isla Martín García y la ribera platense, hasta Punta Indio (Pochettino \& Hurrell, 2013). El trabajo de campo fue realizado en los partidos de Magdalena y Punta Indio, Provincia de Buenos Aires, Argentina. En el Partido de Magdalena, el área de estudio abarca la ciudad de Magdalena (11.093 habitantes), la ciudad de General Mansilla (2.022), Atalaya (720), el barrio Los Naranjos (115) y el paraje La Capilla (sin datos). En el partido de Punta Indio se trabajó en la localidad de Punta del Indio (569) (INDEC, 2010) (Fig. 1A).

Desde el punto de vista fitogeográfico, el área de estudio está situada en el borde oriental de la Provincia Pampeana propuesta por Cabrera (1971), caracterizada por la preponderancia de pastizales o estepas graminosas que se extienden sobre campos altos, con suelos húmicos y arcillo-arenosos ligeramente ácidos (Abba et al., 2009). En el sector costero existe una formación vegetal particular, conocida como "talar" o "bosque de tala" (Fig. 1B), que posee elementos florísticos afines a la Provincia Fitogeográfica del Espinal (Cabrera, 1971; Burkart et al., 1999). La formación se desarrolla a partir del suelo constituido sobre los cordones de conchilla existentes en este sector, con disposición paralela y subparalela a la línea de costa, cuya conformación estuvo vinculada a los cambios en el nivel del mar ocurridos durante el Holoceno (Cavallotto, 2009). Dichos cordones determinan la presencia de ondulaciones características del terreno en este paisaje costero. El talar presenta ocho especies leñosas definitorias, asociadas con gramíneas, plantas trepadoras y rastreras. Las especies leñosas son: Celtis ehrenbergiana (Klotzch) Liebm., "tala” (Celtidaceae), Scutia buxifolia Reissek, "coronillo" (Rhamnaceae), Jodina rhombifolia (Hook. \& Arn.) Reissek, "sombra de toro" (Santalaceae), Schinus longifolius (Lindl.) Speg. longifolius, "molle" (Anacardiaceae), Sambucus australis Cham. \& Schltdl., "sauco" (Caprifoliaceae), Sapium haematospermum Müll. Arg., "curupí" (Euphorbiaceae), Phytolacca dioica L. "ombú" (Phytolaccaceae) y Colletia spinosissima J.F. Gmel., "brusquilla" (Rhamnaceae).

Los bosques de tala alternan con pastizales en zonas altas y con vegetación hidrófila en las zonas bajas. El terreno desciende hacia la costa del río de la Plata y aparece la vegetación de pajonales mixtos, espartillares, pastizales salinos y juncales (Fig. 1C). Por otro lado, se observa en el área la presencia de numerosas especies vegetales exóticas, muchas de estas naturalizadas, como Laurus nobilis L., "laurel" (Lauraceae) y Morus alba L., "morera" (Moraceae), y algunas invasoras: Gleditsia triacanthos L., "acacia negra" (Fabaceae) y Ligustrum lucidum W.T. Aiton, "ligustro" (Oleaceae) (Delucchi \& Torres Robles, 2009). La variedad de comunidades que contienen elementos exóticos conforman un paisaje que da cuenta de una prolongada historia de ocupación humana desde tiempos prehistóricos (Paleo \& Pérez Meroni, 2010). 

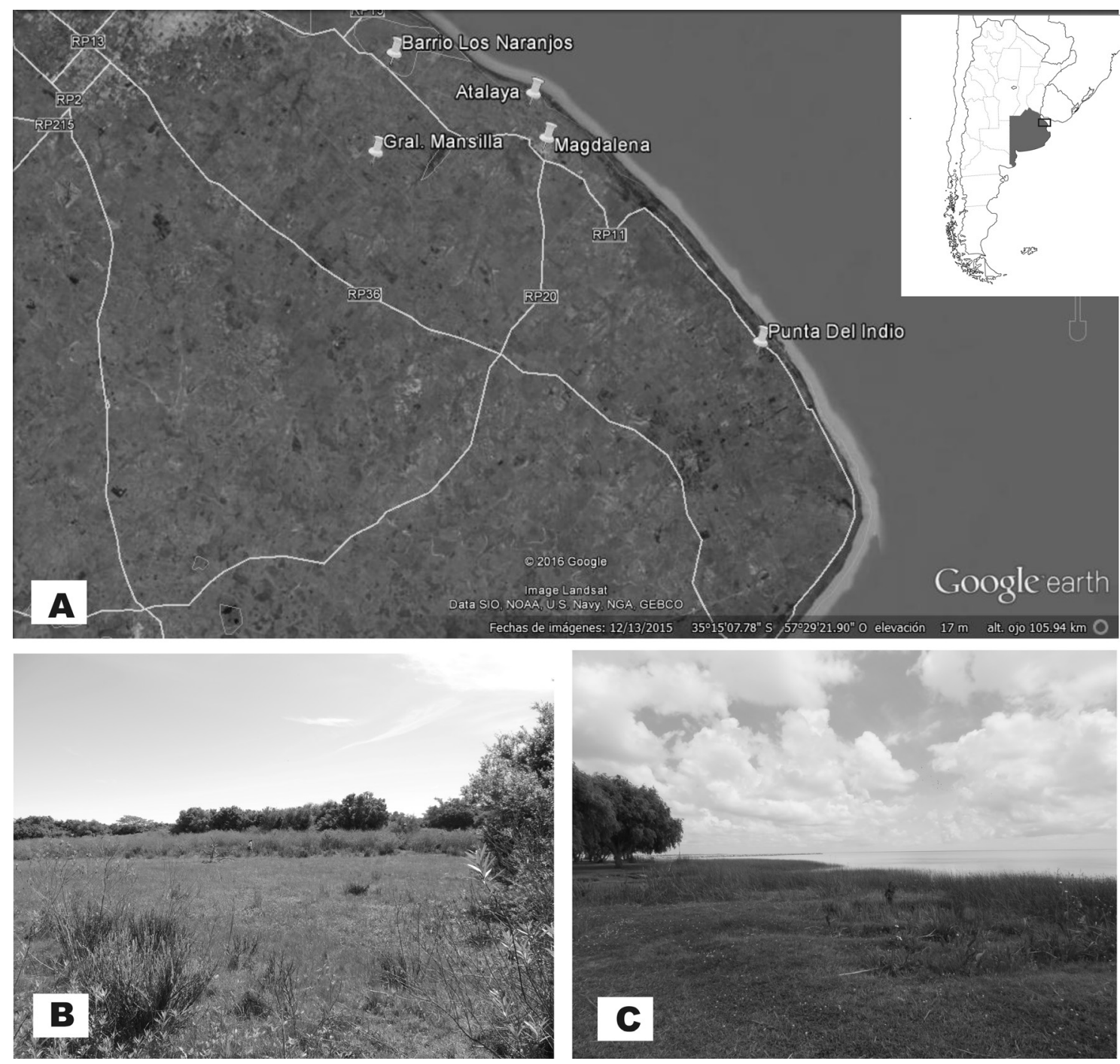

Fig. 1. Área de estudio. A: Mapa de la República Argentina donde se señala la provincia de Buenos Aires y en mayor detalle el noreste de la misma donde se muestran las localidades visitadas. B: Talar en la localidad de Magdalena. C: Juncal en la localidad costera de Atalaya.

Los partidos de Magdalena y Punta Indio contienen sectores netamente urbanos (Magdalena y General Mansilla) y sectores rurales como el paraje La Capilla (General Mansilla). Además, se visitaron enclaves con características intermedias entre la ciudad y el campo (menos de 2000 habitantes, edificación irregular y discontinua, calles de ripio y escasos servicios públicos). Estos sectores corresponden al ámbito "periurbano", definido como un área de transición entre el campo y la ciudad, con predominio de lo urbano
(Atalaya y Punta del Indio) y el rururbano, donde predomina lo rural (Los Naranjos, Estación empalme Magdalena) (Cardoso \& Fritschy, 2012). Las principales actividades económicas son la actividad agropecuaria, la explotación de conchilla y el turismo. También son importantes las prácticas extractivas que se tratan a continuación: extracción de leña y de juncos, para diversos fines.

Asimismo, en esta área se encuentra la Reserva de Biosfera "Parque Costero del Sur" (PCS), creada en el año 1984 por constituir un 
valioso patrimonio natural y cultural en la zona. El PCS abarca una pequeña porción de la costa del Partido de Magdalena y el 70 \% del Partido de Punta Indio. Consta de una franja de $5 \mathrm{~km}$ de ancho por $70 \mathrm{~km}$ de largo sobre el margen sur del río de La Plata, y presenta una superficie de 25.000 hectáreas, formada en su mayoría por propiedades privadas. Es una reserva de protección parcial, categoría VI UICN (Unión Internacional para la Conservación de la Naturaleza), y permite la utilización sostenible de los ecosistemas naturales; sin embargo, existe normativa municipal que prohíbe la total utilización de algunas especies arbóreas nativas.

Parte del área de estudio fue afectada a finales del año 1999 por un derrame de petróleo, producto de la colisión entre un buque tanque de la empresa Shell que ingresaba a uno de los canales del río de La Plata y un buque portacontenedores que se dirigía en dirección contraria. A causa del choque, se derramaron $4.600 \mathrm{~m}^{3}$ de petróleo crudo. La mancha de petróleo afectó $23 \mathrm{~km}$ de humedales y dos áreas de recreación, Playa Nueva y Balneario Magdalena. Una vez que el derrame llegó a las costas del Partido de Magdalena, se vieron afectados de forma directa un conjunto de actores sociales (junqueros, pescadores, dueños de campings y proveedurías) y el mismo municipio de Magdalena, al perder la actividad turística (Acerbi \& Barrenechea, 1999).

\section{Aspectos metodológicos}

El trabajo de campo fue realizado durante 11 viajes de campaña entre 2015 y 2016. Previo consentimiento informado, se realizó un total de 24 entrevistas abiertas y semiestructuradas, tanto individuales como grupales, a personas de ambos sexos, entre 40 y 75 años de edad, oriundos de la zona. Asimismo, se implementó la observación participante y se efectuaron caminatas con los informantes por los diferentes espacios transitados en el desarrollo de las actividades estudiadas (Cotton, 1998). Del total de informantes, 14 realizan sólo la actividad leñatera, 6 sólo la actividad junquera, y 4 ambas prácticas. Además, se ha empleado la técnica de análisis de "historias de vida", con el fin de indagar en la relación entre los pobladores locales y el ambiente de manera retrospectiva hacia décadas relativamente recientes: mediados de siglo XX (Trinidade Medeiros et al., 2014).

Para la selección de informantes calificados (personas consideradas localmente como vinculadas con las actividades mencionadas tanto en la actualidad como en el pasado) se empleó el muestreo de "bola de nieve" (Bernard, 2000). Se entrevistaron junqueros (cortadores, vendedores y artesanos del junco) y leñateros (consumidores a nivel doméstico y también dos ladrilleras aún en funcionamiento en Magdalena y vendedores de leña). En las entrevistas se exploraron dos ejes temáticos: 1 . Los conocimientos relacionados a la actividad junquera o leñatera según correspondiera (especies empleadas, preferencias, usos, prácticas sobre las mismas, calendario de actividades/época de cosecha y los sitios de colecta) y 2. La evaluación de los cambios ambientales, cambios en la disponibilidad del recurso, en la distancia recorrida y reemplazo de las especies preferidas por otras.

La información fue registrada con grabadora y se tomaron fotografías con cámara digital. Se realizaron caminatas con algunos informantes a fin de identificar las especies que utilizan comúnmente y se tomó una muestra de cada planta mencionada como material de referencia, depositado en el Laboratorio de Etnobotánica y Botánica Aplicada (LEBA). Mediante el empleo de material bibliográfico se llevó a cabo la identificación botánica del material vegetal, así como las características de las maderas (Tortorelli, 1956; Cabrera \& Zardini, 1978; Hurrell, 2004). Para el tratamiento sistemático de las especies de plantas involucradas, nativas y exóticas, se emplearon las bases de datos de IBODA (2016) y The Plant List (2016), respectivamente.

\section{Resultados y Discusión}

\section{Leñateros}

Actualmente, 16 de los 18 entrevistados utilizan leña. La obtienen mediante la recolección directa y además, en 5 casos, se registró un complemento mediante la compra de leña. Los 2 entrevistados que no utilizan 
leña en la actualidad, igualmente fueron incluidos en los resultados debido a que uno de ellos fue vendedor de leña hasta hace pocos años, y el otro actualmente vende leña pero, al contar con gas natural, ya no utiliza este recurso. El principal uso es para la calefacción de los hogares durante el invierno (salamandra, estufa hogar a leña) $\mathrm{y}$, en menor medida, para cocinar (asados, cocina de leña o de barro). Asimismo, este recurso es muy valorado por dos ladrilleras de General Mansilla para la cocción de ladrillos en los hornos (Fig. 2A).

Se registró un total de 23 especies leñosas utilizadas como leña (Tabla 1). El género mencionado con más frecuencia fue Eucalyptus L'Héritier (Myrtaceae), con un total de 16 menciones: "eucalipto medicinal" (E. globulus Labill.) (3), "eucalipto colorado" (E. camaldulensis Dehnh.) (6) y "eucalipto" sin distinción de especies (Eucalyptus spp.) (7). Luego figuran el "tala" (Celtis ehrenbergiana) y el "coronillo" (Scutia buxifolia) con 11 y 7 menciones, respectivamente. Sin embargo, al preguntar por las especies preferidas, la mayoría de los entrevistados indicaron estas dos últimas especies nativas, pero sostienen que no se pueden "cortar" en la reserva (PCS) y que el "coronillo" sólo se encuentra allí. En Punta del Indio, todos los entrevistados mencionaron que está prohibido el uso de especies nativas, mientras que en Magdalena sólo 4 personas comentaron que en la costa no se puede "cortar". Al respecto, un informante de Magdalena dijo: "prefiero tala y coronillo, pero en la reserva no se puede juntar, y acá no hay coronillo; tala sí, algo hay". Ambas especies son valoradas como "buena leña" por la duración de sus brasas y el calor que generan, precisamente son de las especies mencionadas en las entrevistas las maderas de mayor densidad, las más duras y pesadas según la bibliografía (Tortorelli, 1956). El 89\% de los entrevistados mencionó la recolección o compra de especies exóticas como leña. El uso del "eucalipto" resulta una buena alternativa debido a que "hace buena llama y se consigue fácilmente", ya que en la zona abundan los montes implantados. Asimismo, todos los informantes mencionaron que complementan el uso de leña con el uso de gas para cocinar y calefaccionar los hogares. A partir de 2011, las localidades de Magdalena, General Mansilla y Atalaya disponen de gas natural, mientras que en Los Naranjos, Paraje La Capilla, Estación empalme Magdalena y Punta del Indio se utiliza gas envasado (garrafa). Sólo 4 de las unidades domésticas visitadas cuentan con gas natural.

La época de aprovisionamiento de leña es principalmente el invierno. Los vendedores suelen comenzar a cortar un tiempo antes para tener un suministro de leña seca. La recolección es llevada a cabo por personas de ambos sexos y de diferentes edades, desde niños hasta ancianos, y en el caso de las podas y extracciones sólo las realizan hombres
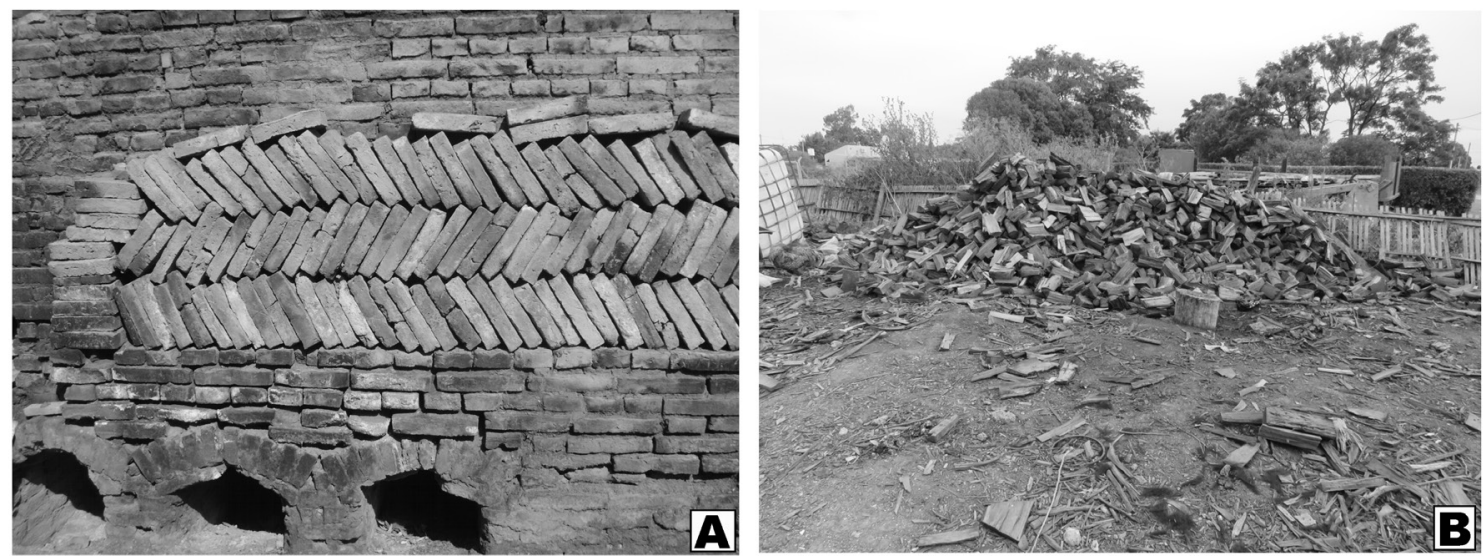

Fig. 2. Actividad leñatera. A. Horno de ladrillo en la localidad de Magdalena. B: Pila de leña de eucalipto para venta en Magdalena. 
Tabla 1. Especies leñateras utilizadas por los entrevistados.

\begin{tabular}{|c|c|}
\hline Nombre científico y local & Familia \\
\hline Casuarina cunninghamiana Miq. (Casuarina) & Casuarinaceae \\
\hline Celtis australis L. (Almez) & \\
\hline Celtis ehrenbergiana (Klotzsch) Liebm. (Tala) & \\
\hline Acacia caven (Molina) Molina (Espinillo) & \multirow{5}{*}{ Fabaceae } \\
\hline Acacia melanoxylon R. Br. (Acacia melanosa) & \\
\hline Gleditsia triacanthos L. (Acacia negra) & \\
\hline Parkinsonia aculeata L. (Cina cina) & \\
\hline Robinia pseudoacacia L. (Acacia blanca) & \\
\hline Laurus nobilis L. (Laurel) & Lauraceae \\
\hline Melia azedarach L. (Paraíso) & Meliaceae \\
\hline Morus alba L. (Morera) & Moraceae \\
\hline Eucalyptus camaldulensis Dehnh (Eucaliptus colorado) & \multirow{3}{*}{ Myrtaceae } \\
\hline Eucalyptus globulus Labill. (Eucalipto medicinal) & \\
\hline Eucalyptus spp. (Eucalipto) & \\
\hline Fraxinus excelsior L. (Fresno) & \multirow[t]{2}{*}{ Oleaeceae } \\
\hline Ligustrum lucidum W. T. Aiton (Ligustro) & \\
\hline Pinus spp. (Pino) & Pinaceae \\
\hline Colletia spinosissima J. F. Gmel. (Brusquilla) & \multirow{2}{*}{ Rhamnaceae } \\
\hline Scutia buxifolia Reissek (Coronillo) & \\
\hline Prunus domestica L. (Ciruelo) & Rosaceae \\
\hline Salix spp. (Sauce) & \multirow{2}{*}{ Salicaceae } \\
\hline Populus spp. (Álamo) & \\
\hline
\end{tabular}


mayores. La mayoría recolecta ramas secas de los alrededores de las casas, o también recorren distancias mayores, hacia la costa. Asimismo, se registró el aprovechamiento de residuos de podas municipales, la extracción de árboles y ramas caídas, podas en altura, tala de montes de "eucalipto" de campos cercanos y el aprovechamiento de la leña producto de la "limpieza" de terrenos para construir. La frecuencia de abastecimiento varía: semanal, mensual o para todo el invierno, conformando pilas de leña (Fig. 2B), transportándola caminando, en camioneta o en camión. El corte se realiza mediante motosierras, hachas y sierras sin fin, con frecuencia, las ramas se trozan para luego obtener "astillas".

Como lo sugiere el estudio de Cardoso (2012) para el noroeste de la Patagonia, se puede observar el valor que localmente se le da al recurso leñatero, la vigencia de su uso y el conocimiento que orienta la selección y las prácticas asociadas al mismo.

\section{Junqueros}

El $80 \%$ de los entrevistados se dedicaron a la actividad junquera en algún momento de su vida, excepto dos artesanos que siempre compraron el junco ya seco como materia prima para elaborar sus artesanías. Sin embargo, en la actualidad, sólo uno de los entrevistados continúa dedicándose de forma secundaria a esta actividad. A su vez, sólo un informante pertenece a una familia que vivió exclusivamente de cortar juncos; el resto de los entrevistados complementaban la actividad con otros trabajos (comercio, pesca, artesanato).

La recolección, se realiza desde fines del invierno o principios de la primavera hasta fines del verano o principios del otoño, época en que las plantas alcanzan la altura suficiente para su utilización $(1,60 \mathrm{~m})$ y en la que las condiciones meteorológicas permiten su secado. Todos los entrevistados relataron que en invierno es preferible no cortar; en primer lugar, para evitar el frío; en segundo lugar, porque dicen que el junco es de mala calidad; incluso, algunos informantes narran que si se corta en invierno el junco no se recupera. Sin embargo, algunos junqueros expresaron que en las "buenas épocas" (década de 1980) cortaban todo el año de forma ininterrumpida. Los juncos son cortados al ras del suelo con hoces fabricadas artesanalmente por ellos mismos, utilizando como material base guadañas y cuchillos, o bien partes de estos, aunque también eran compradas (Fig. $3 \mathrm{~A})$. El corte se realiza con el río bajo y el sustrato al descubierto, para evitar accidentes. Con los juncos recién cortados se forman "brazadas" (todos los juncos que se pueden apilar y sujetar con ambos brazos), y se arman las balsas, disponiéndose de forma aleatoria las partes gruesas y finas de los juncos, y se espera la crecida del río para llevarlos a tierra firme o, en caso de contar con caballos, se los transportan a tiro. Una vez en el hogar, las
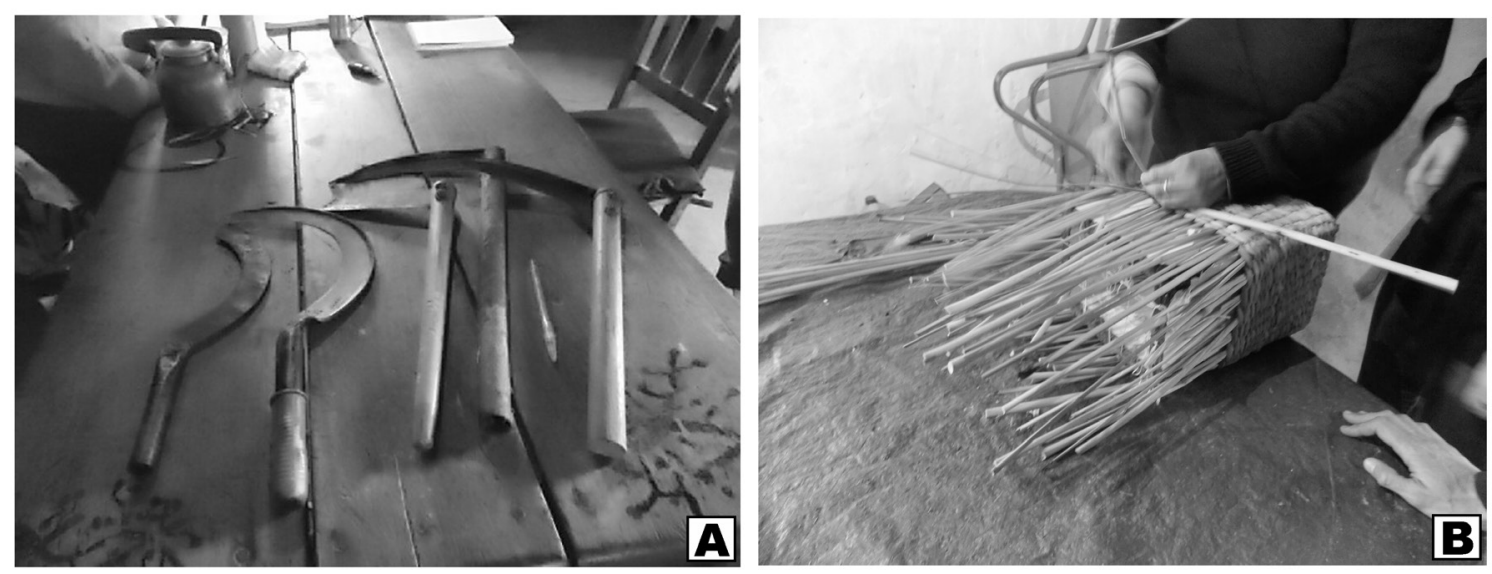

Fig. 3. Actividad junquera. A: Herramientas utilizadas por una familia junquera. B: Artesana tejiendo una canasta con junco. 
"brazadas" son "limpiadas" sacudiéndolas desde la parte media de forma que caigan los juncos secos y de menor longitud. Cada brazada es dividida en tres "mazos" (todos los juncos que pueden sujetar con ambas manos) $\mathrm{y}$ atados con el mismo junco. Durante todo este proceso el junco es considerado "verde" $\mathrm{y}$ algunos junqueros lo venden en ese estado. Otros realizan el secado, que le agrega valor y aclara el junco, disponiendo los "mazos" al sol en forma de abanico, en terrenos amplios, volteándolos de ambos lados para secarlos.

Los tipos de juncos reconocidos fueron tres: "redondo" o "liso", de sección transversal circular y coloración verde oscura; "triangular" o "rango", de sección transversal triangular y coloración similar al anterior; y "cebollita", mucho más pequeño que los anteriores, de menor diámetro y coloración verde clara. El primero se utiliza en las huertas para atar plantas de tomate, morrón y berenjena a las respectivas cañas. Era el tipo de junco más recolectado hace décadas, debido a la alta demanda de las "quintas" del Cinturón Hortícola Platense; incluso, era vendido en Mar del Plata. Esta práctica fue reemplazada por el hilo de polietileno con la llegada de los invernáculos. El junco "triangular" se utiliza para artesanías rústicas, como esteras y canastas. El "cebollita", más delicado, sirve tanto para la huerta (para atar plantas de ajo y cebolla) como para fabricar artesanías de tejidos más finos. La variedad "triangular" pertenece a la especie Schoenoplectus californicus, mientras que las otras dos no pudieron ser identificadas hasta el momento, debido a la ausencia de estructuras reproductivas en los períodos de muestreo. El junco "redondo" podría corresponder a $S$. tabernaemontani (C.C. Gmel.) Palla, citado para la zona (Cabrera \& Zardini, 1978). Sin embargo, no se descarta que los tres tipos pertenezcan a diferentes estadios o fenotipos de la misma especie.

Tres de las personas entrevistadas realizaron alguna vez artesanías en junco. Uno de ellos es un junquero tradicional de la zona que se dedicaba a reparar muebles, entre otros trabajos. Las otras dos personas son mujeres, una de ellas proveniente de una familia de tradición junquera; la otra, no. Sin embargo, ambas aprendieron la profesión de la misma persona, y venden sus artesanías como un ingreso extra, porque no viven de esa actividad (Fig. 3B). Estudios realizados en Ecuador y Perú, mencionan otros usos para los juncos (denominados en esa zona "totora"): alimentarios (los rizomas) y para la construcción de las casas-embarcaciones donde viven los pobladores locales (Heiser, 1979).

\section{Cambio ambiental}

Los diferentes tópicos del cambio ambiental relevados en la narrativa de los locales están resumidos en la Tabla 2 .

Con respecto a la actividad leñatera, una única persona en Magdalena mencionó que "hay menos monte ahora, nadie planta montes nuevos y todos tienen salamandra". Esto contrasta con lo señalado por Cardoso et al. (2012) para la Patagonia, quienes registraron que la totalidad de los informantes, año tras año, deben recorrer mayores distancias para encontrar la leña caída de las especies preferidas, hecho que evidencia una escasez creciente del recurso, por lo que se complementa con la compra y uso de combustibles alternativos. Para nuestra área de estudio, el resto de los entrevistados indicaron que encuentran fácil la leña que necesitan, no tienen que caminar más que antes, no hace falta usar estiércol, raíces, ni cardos secos, aunque saben que queman bien, porque hay suficiente leña disponible. Al respecto, un entrevistado en Los Naranjos dijo: "conseguimos fácil la leña, ahora hay más árboles que antes en el barrio", y utilizó como elemento de prueba fotografías de la zona de hace 30 años, donde se veían algunos pocos árboles dispersos en una matriz de pastizal. Para el caso de Punta Indio, la dificultad reside en la prohibición de extracción más que en la falta del recurso, por eso se recurre a la compra de leña o se utilizan especies exóticas como "acacia negra", "laurel" o "ligustro" que se pueden cortar sin problemas en la reserva. Se relevaron diferentes estrategias para la conservación del talar en las entrevistas con los pobladores que viven dentro del PCS, lo que implica un cambio en la valoración del mismo. Un informante expresó claramente: "mi padre vivió toda la vida peleando contra el tala. Esa era la concepción que había de antes del tala, 
Tabla 2. Cambios ambientales.

\begin{tabular}{|l|l|}
\hline \multicolumn{1}{|c|}{ Junqueros } & \multicolumn{1}{|c|}{ Leñateros } \\
\hline $\begin{array}{l}\text { Cambios en la costa producto del derrame de } \\
\text { petróleo (afectó el agua y los sedimentos y, con } \\
\text { ello, la cantidad y calidad de juncos presentes y la } \\
\text { fauna). }\end{array}$ & Mayor cantidad de montes implantados. \\
\hline $\begin{array}{l}\text { Cambios en los sistemas productivos (con el } \\
\text { invernáculo se dejó de utilizar el junco para encañar } \\
\text { plantas hortícolas). }\end{array}$ & $\begin{array}{l}\text { Cambios en la legislación en torno a la creación } \\
\text { del PCS. }\end{array}$ \\
\hline $\begin{array}{l}\text { Disminución de la cantidad de juncos por la } \\
\text { actividad ganadera. }\end{array}$ & Cambios en la composición arbórea. \\
\hline $\begin{array}{l}\text { Aumento de la cantidad de juncos por la presencia } \\
\text { de caballos. }\end{array}$ & $\begin{array}{l}\text { Uso de especies exóticas en reemplazo de las } \\
\text { nativas. }\end{array}$ \\
\hline $\begin{array}{l}\text { Erosión de las costas versus depositación de } \\
\text { sedimentos. }\end{array}$ & Cambios en la valoración del talar. \\
\hline \multicolumn{1}{|c|}{ Ambos actores sociales } \\
\hline Cambios en la abundancia de determinadas especies de animales. \\
\hline
\end{tabular}

había que eliminar el tala, eliminarlo, era como los indios".

Los talares bonaerenses han sufrido un proceso de degradación muy importante producto de prácticas de manejo no sustentables. Las referencias históricas apuntan a un uso intensivo de la madera del "tala" desde comienzos de la ocupación hispánica bonaerense, en el siglo XVI, principalmente como forma de combustible y para la construcción de viviendas y cercos (Athor, 2006; Torres Robles \& Tur, 2006). Actualmente, las principales actividades económicas (la ganadería y la extracción de conchilla del sustrato) en los campos privados que componen el PCS, afectan la estructura y superficie de los bosques, la composición florística y el relieve. Arturi et al.
(2006) plantean la posibilidad del desarrollo de sistemas silvopastoriles en los talares, como forma de integrar al bosque al esquema productivo de la zona. Asimismo, la tala indiscriminada ha llevado a una reducción notoria de los talares en la actualidad (Torres Robles \& Arturi, 2009). Sin embargo, al igual que comentan Richeri et al. (2013) para la Patagonia, es posible observar en el área de estudio soluciones locales y flexibilidad en el conocimiento y en las prácticas sobre el uso de vegetales. Ante la legislación en torno al PCS, los pobladores locales establecieron diferentes estrategias para reemplazar las especies preferidas como leña, pero que ya no pueden utilizar, mediante la diversificación de especies empleadas, incluyendo diversas plantas leñosas exóticas. Estas prácticas podrían colaborar 
con las actividades de control de exóticas invasoras que se vienen realizando por propia iniciativa de los pobladores y de la asociación civil "Amigos del PCS", con el apoyo del Municipio de Punta del Indio. Asimismo, es posible observar un máximo aprovechamiento del recurso leñatero, en la mayoría de los casos utilizando ramas secas y árboles muertos.

Otra estrategia ampliamente utilizada es la compra de leña y el uso de gas. De las cuatro unidades domésticas que disponen de gas natural, dos de estas siguen eligiendo utilizar también leña, y el resto de los entrevistados que emplean garrafa, probablemente debido al elevado costo de la misma, complementan el uso de gas con el de leña. A 32 años de la creación del PCS, es posible observar un cambio en la valoración del talar: la mayoría de los leñateros mencionaron que está prohibido utilizar ciertas especies nativas dentro del parque, en contraposición a la postura de erradicar el "tala" que existía antiguamente.

Con respecto a la actividad junquera, los informantes evaluaron como cambios ambientales el efecto negativo del derrame de petróleo del año 1999 sobre los juncales y la actividad junquera (y, consecuentemente, sobre la economía familiar), así como los cambios en las actividades productivas regionales que afectaron a la actividad junquera y la erosión de las costas por el propio río (Tabla 2). La totalidad de los entrevistados señalaron un antes y un después del derrame de petróleo. A partir de este siniestro, la actividad junquera se vio perjudicada, reconociéndose en la actualidad los daños en los sedimentos del río, en los juncales y, fundamentalmente, en la memoria y los sentimientos de los junqueros. En un primer momento, luego del derrame, se les prohibió continuar con la actividad extractiva. Todos los entrevistados cuentan que el petróleo "quemó" el junco, mientras que algunas plantas no murieron pero los tallos quedaron "manchados" por el petróleo, disminuyendo su calidad y propiedades plásticas. La totalidad de los informantes atribuyó la disminución del junco al derrame de petróleo, por muerte de los rizomas.

Por otra parte, el cambio en las actividades productivas regionales, principalmente la hortícola, influyó de forma marcada en el comercio del junco, hecho mencionado por el $50 \%$ de los informantes. La mayor parte de la materia prima era vendida a los productores hortícolas de La Plata y de Mar del Plata, para atar las plantas (en especial, de tomate) a las cañas en los cultivos al aire libre. La producción bajo cubierta (invernáculos) implicó dejar de utilizar juncos, con lo cual declinó abruptamente la demanda. A su vez, la actividad ganadera también influyó en la disponibilidad de los juncales: el 20\% de los informantes relacionaron la disminución de los juncales con la cría intensiva de ganado vacuno en las costas, en especial durante el invierno, cuando escasean las pasturas. Sin embargo, un informante vinculó el aumento de los juncales con la presencia de ganado equino, en particular en Punta Blanca (en el límite de Magdalena con Berisso), incremento que seguramente estuvo relacionado a la prohibición del ingreso a la costa por varios años (desaparición del estudiante Miguel Bru en la década de 1990).

Aunque parece que el derrame de petróleo de 1999 fue el principal desencadenante del ocaso de la actividad junquera, al analizar los cambios de los sistemas productivos en el Cinturón Hortícola Platense (CHP) se observa que esta actividad extractiva ya estaba en retroceso. La adopción del cultivo bajo cubierta comenzó en la década de 1980 y logró establecerse definitivamente hacia la de 1990, logrando un crecimiento exponencial luego de la recesión de 1998-2001, hasta alcanzar en el presente más del 79\% de la superficie total cubierta por invernáculos en el CHP (García, 2011, 2012). Este sistema de cultivo permitió mayor productividad al lograr ambientes controlados, lo que significó la inutilidad de los juncos y la llegada de más insumos plásticos para soporte de las plantas de tomates, berenjenas y morrones, entre otras. La mayor frecuencia de referencias del derrame de petróleo con respecto al cambio en las actividades productivas regionales de las narrativas puede explicarse mediante el concepto de "memoria de perturbación". En el sentido de Arce-Nazario (2007), la memoria de perturbación se crea al eliminarse el componente humano del ambiente, reflejada -en este caso- en la totalidad de las narrativas 
de los informantes. Sin embargo, el cambio en la producción hortícola, parece ser la causa principal del decrecimiento de la actividad, dado que hoy en día la recolección de juncos está relacionada en un porcentaje bajo con la obtención de materia prima para la elaboración de artesanías.

Otro cambio reconocido, relacionado a la disminución de los juncales, es la erosión de las costas en determinadas áreas, sólo un informante destacó el depósito en otras. La costa del río constituye un ambiente muy variable. En Atalaya, un informante comentó que "hace 15-20 años desde la escollera veías la gente que se bañaba en Magdalena. De balneario a balneario se veía todo. Cambió todo". Los pulsos de avance y retroceso de las zonas urbanizadas, y cultivadas respecto de la vegetación espontánea mencionados por Hurrell \& Delucchi (2013) y Pochettino \& Hurrell (2013) para otros sectores de la ribera rioplatense, constituyen un proceso importante en la modelización del paisaje local que deben ser tenidos en cuenta en el estudio del cambio ambiental y en la ecología de las invasiones biológicas.

Los dos grupos de actores sociales (leñateros y junqueros) mencionaron además cambios en la abundancia de determinadas especies y en las condiciones climáticas. Al respecto, se remarcó la mayor cantidad de "lagartos" (Tupinambis sp. Daudin, 1802, Teiidae), "cotorras" (Myiopsitta monachus Boddaert, 1783, Psittacidae), "paloma grande" (Columba picazuro Temminck, 1813, Columbidae), anfibios (sapos y ranas), bivalvos (almejas), como también la disminución poblacional de "tucu-tucu" (Ctenomys sp. Blainville, 1826, Ctenomyidae) y "víboras", exceptuando las "yararás" asociadas a los "camalotes" (Eichhornia crassipes [Mart.] Solms, Pontederiaceae) que llegan del litoral platense con las crecidas. En relación al clima, se mencionaron algunos cambios tales como inviernos menos fríos, con heladas de menor intensidad, y mayor humedad. Estos últimos cambios pueden relacionarse al avance de la urbanización que condiciona la abundancia de algunos taxa en detrimento de otros (Knapp et al., 2008; Kühn et al., 2004), como también en el efecto en la temperatura que produce altas concentraciones de cemento, emisiones de gases, generación de calor, entre otros.

En concordancia con los trabajos de Vitousek (1992), Parlee et al. (2012), ArceNazario (2007) y Celentano et al. (2014), el cambio ambiental incluye dimensiones sociales como los cambios en el uso de la tierra, la deforestación, la extracción minera, la contaminación atmosférica y problemas sociales como el alcoholismo. Asimismo, los resultados contrastan con los obtenidos por Pochettino \& Hurrell (2013) para la Isla Paulino (Partido de Berisso), donde la narrativa de los pobladores destaca los cambios climáticos como principales emergentes de los cambios ambientales. Esto puede relacionarse al efecto devastador que tienen las crecidas del río de la Plata en las zonas costeras sobre las huertas de los pobladores, difiriendo del efecto que pueden tener sobre un juncal o cultivo de frutales perennes.

\section{Conclusiones}

Con anterioridad a la creación del Parque Costero del Sur, la actividad leñatera se vio favorecida por la extracción de leña de los talares y fomentada, a su vez, por la explotación de conchilla del sustrato donde el talar se desarrolla y, por otro lado, por la implantación de bosques de eucaliptos. Actualmente, debido a la prohibición de la primera actividad, ligada a las gestiones conservacionistas en el marco del PCS, la provisión principal se limita a la obtención de leña de los bosques implantados así como también de la vegetación espontánea que permite la legislación. La abundancia del recurso combustible y la reformulación en las prácticas y estrategias de selección hizo posible la permanencia de la actividad a pesar de los cambios sufridos en el área de estudio.

Por el contrario, la actividad junquera cesó por dos causas principales, una "silenciosa", esto es, el cambio en la producción hortícola regional, que en la actualidad sigue condicionando dicha actividad, y un componente más explícito: el derrame de petróleo.

Asimismo, las actividades realizadas por los informantes, la leñatera por un lado, 
la junquera por el otro, influyeron en la valoración del cambio ambiental local, dado que se observaron algunos pocos cambios en común, principalmente en relación al clima y a la fauna, mientras que los cambios más importantes registrados fueron en relación a los recursos utilizados y a las actividades en torno a los mismos.

\section{Agradecimientos}

A los pobladores de Punta Indio y Magdalena que han colaborado con el desarrollo de este trabajo y han autorizado la difusión de los resultados. A los revisores, por sus oportunos comentarios y sugerencias. A la Dra. M. Lelia Pochettino y al Dr. Julio A. Hurrell por la lectura crítica del manuscrito y las sugerencias que lo enriquecieron. Este estudio se realizó con el apoyo financiero de FONCyT (PICT 2539), CONICET (PIP 0460) y UNLP (Proyecto de Incentivos N-715).

\section{Bibliografía}

ABBA, A. M., M. L. MERINO \& S. F. VIZCAÍNO. 2009. Mamíferos del Parque Costero del Sur: caracterización general y un ejemplo de trabajo. En J. ATHOR (ed.), Parque Costero del Sur. Naturaleza, conservación y patrimonio cultural, pp. 172-199. Fundación de Historia natural Félix de Azara, Buenos Aires.

ACERBI, M. \& J. BARRENECHEA. 1999. [Última actualización 2006, consulta 2016]. PIRNA: Análisis de las estrategias frente al derrame de petróleo en Magdalena (Provincia de Buenos Aires, Argentina). Programa de Investigaciones en Recursos Naturales y Ambiente, http://www.pirna.com.ar/files/pirna/ PON-Acerbi-Barrenechea-Derrame $\% 20$ en $\% 20$ Magdalena.pdf.

ARCE-NAZARIO, J. A. 2007. Landscape images in Amazonia narrative: The role of oral history in environmental research. Conservat. Soc. 5: 115-133.

ARTURI, M. F., C. A. PÉREZ, M. HORLENT, J. F. GOYA \& S. S. TORRES ROBLES. 2006. El manejo de los talares de Magdalena y Punta Indio como estrategia para su conservación. En E. MÉRIDA \& J. ATHOR (eds.), Talares Bonaerenses y su Conservación, pp 37-45. Fundación de Historia Natural Félix de Azara, Buenos Aires.

ATHOR, J. 2006. Referencias bibliográficas históricas que delatan la presencia del talar en la ciudad de
Buenos Aires. En E. MÉRIDA \& J. ATHOR (eds.), Talares bonaerenses y su conservación, pp. 218222. Fundación de Historia Natural Félix de Azara. Buenos Aires.

BERNARD, R. H. 2000. Social Research Methods. Qualitative and Quantitative Approaches. Sage, Thousand Oaks. 659 pp.

BURKART, R., N. O. BÁRBARO, R. O. SÁNCHEZ \& D. A. GÓMEZ. 1999. Eco-regiones de la Argentina. Administración de Parques Nacionales, Buenos Aires. 43 pp.

CABRERA, A. L. 1971. Fitogeografía de la República Argentina. Bol. Soc. Argent. Bot. 14: 1-42.

CABRERA, A. L. \& E. M. ZARDINI. 1978. Manual de la flora de los alrededores de Buenos Aires. Editorial ACME, 2da. edición. 589 pp.

CARDOSO, M. B. 2012. Utilización de especies combustibles en comunidades locales del noroeste de Patagonia: Bienes culturales y ambientales en la subsistencia rural. Tesis doctoral, Centro Regional Universitario Bariloche, Universidad Nacional del Comahue. 166 pp.

CARDOSO, M. B., A. H. LADIO \& M. LOZADA. 2012. The use of firewood in a Mapuche community in a semi-arid region of Patagonia, Argentina. Biomass Bioenerg. 46: 155-164.

CARDOSO, M. M. \& B. A. FRITSCHY. 2012. Revisión de la definición del espacio rururbano y sus criterios de delimitación. Contrib. Científ. 241: 27-39.

CARROLL, C., M. K. PHILLIPS, N. H. SCHUMAKER \& D. W. SMITH. 2003. Impacts of landscape change on wolf restoration success: Planning a reintroduction program based on static and dynamic spatial models. Conserv. Biol. 17: 536-548.

CAVAllotTO, J. L. 2009. Caracterización e historia evolutiva del sustrato sobre el que se desarrollan los talares del nordeste bonaerense. En J. ATHOR (ed.), Parque Costero del Sur. Naturaleza, conservación y patrimonio cultural, pp. 66-81. Fundación de Historia natural Félix de Azara, Buenos Aires.

CELENTANO, D., G. X. ROUSSEAU, V. L. ENGEL, C. L. FAÇANHA, E. M. de OLIVEIRA \& E. G. de MOURA. 2014. Perceptions of environmental change and use of traditional knowledge to plan riparian forest restoration with relocated communities in Alcântara, Eastern Amazon. J. Ethnobiol. Ethnomed. 10: 11 .

COTTON, C. M. 1998. Ethnobotany. Principles and Applications. John Wiley \& Sons, New York. 424 pp.

DELUCCHI, G. \& S. S. TORRES ROBLES. 2009. Plantas exóticas en el Parque Costero del Sur: Una categorización. En J. ATHOR (ed.), Parque Costero del Sur. Naturaleza, conservación y patrimonio cultural, pp. 408-415. Fundación de Historia natural Félix de Azara, Buenos Aires.

ETTER, A., C. MCALPINE \& H. POSSINGHAM. 2008. Historical Patterns and Drivers of Landscape 
Change in Colombia since 1500: A Regionalized Spatial Approach. Ann. Assoc. Am. Geogr. 98: 2-23.

GARCÍA, M. 2011. El cinturón hortícola platense: ahogándonos en un mar de plásticos. Un ensayo acerca de la tecnología, el ambiente y la política. Theomai 23: 35-53.

GARCÍA, M. 2012. Análisis de las transformaciones de la estructura agraria hortícola platense en los últimos 20 años. El rol de los horticultores bolivianos. Tesis Doctoral, Facultad de Ciencias Agrarias y forestales, Universidad Nacional de La Plata. 432 pp.

GARCÍA CODRÓN, J. C. \& F. SILIÓ CERVERA. 2000. Riesgos naturales en los Andes: cambio ambiental, percepción y sostenibilidad. B. Asoc. Geógr. Esp. 30: 69-84.

GERRITSEN, P. R., M. MONTERO \& P. FIGUEROA. 2002. El mundo en un espejo. Percepciones campesinas de los cambios ambientales en el Occidente de México. Econ. Soc. Territ. 4: 253-278.

HEISER Jr, C. B. 1979. The totora (Scirpus californicus) in Ecuador and Peru. Econ. Bot. 32: 222-236.

HURRELL, J. A. (ed.) 2004. Árboles rioplatenses. Biota Rioplatense III, Buenos Aires, Editorial L.O.L.A. 300 pp.

HURRELL, J. A. 2014. Urban ethnobotany in Argentina: Theoretical advances and methodological strategies. Ethnobio. Conserv. 3: 2.

HURRELL, J. A. \& U.P. ALBUQUERQUE. 2012. Is Ethnobotany an Ecological Science? Steps towards a complex Ethnobotany. Ethnobio. Conserv. 1: 4.

HURRELL, J. A. \& G. DELUCCHI. 2013. Aportes de la etnobotánica al estudio de las invasiones biológicas. Casos en la región Rioplatense (Argentina). Rev. Hist. Nat. 3: 61-76.

HURRELL, J. A., P. C. STAMPELlA \& A. M. MARTÍNEZ. 2015. Perspectivas etnocientíficas en el estudio del cambio ambiental en la ribera platense, Argentina. Actas VI ICEB, International Congress of Ethnobotany (en prensa). Córdoba, España.

IBODA. 2016. [Permanentemente actualizado, consulta 2016]. Instituto de Botánica Darwinion. Flora Argentina. Disponible: http://www.darwin.edu.ar/.

INDEC, 2010. [Última actualización 2014, consulta 2016]. Instituto Nacional de Estadística y Censos. Censo Nacional de Población, Hogares y Viviendas, 2010, http://www.censo2010.indec.gov.ar.

KNAPP, , S., I. KÜHN, V. MOSBRUGGER \& S. KLOTZ. 2008. Do protected areas in urban and rural landscapes differ in species diversity? Biodivers. Conserv. 17: 1595-1612.

KÜHN, I., R. BRANDL \& S. KLOTZ. 2004. The flora of German cities is naturally species rich. Evol. Ecol. Res. 6: 749-764.

LANE, R. 1997. Oral Histories and Scientific Knowledge in Understanding Environmental Change: a case study in the Tumut Region, NSW. Aust. Geogr. Stud. 35: 195-205.
MAFFI, L. 2001. Introduction. On the interdependence of biological and cultural diversity. En L. MAFFI (ed.), On biocultural diversity. Linking language, knowledge, and the environment, pp. 1-50, Smithsonian Institution Press, Washington.

MANZOLI, D. E., L. R. ANTONIAZZI \& P. M. BELDOMENICO. 2011. Cambio ambiental global, parásitos y la salud de sus hospedadores: las moscas parásitas del género Philornis en pichones de aves. El Hornero 26: 45-53.

NAZAREA, V. (ed.). 1999. Ethnoecology. Situated knowledge/located lives. University of Arizona Press, Tucson. 299 p.

PALEO, M. C. \& M. PÉREZ MERONI. 2010. Del bosque de tala al Parque Costero del Sur. En M. BERÓN, L. LUNA, M. BONOMO, C.MONTALVO, C. ARANDA \& M. CARRERA-AIZPITARTE (eds.), Mamül Mapu: Pasado y presente desde la arqueología pampeana 1, pp. 215-226. Libros del Espinillo, Ayacucho.

PARLEE, B. L., K. GEERTSEMA \& A. WILLIER. 2012. Social-ecological thresholds in a changing boreal landscape: insights from Cree knowledge of the Lesser Slave Lake region of Alberta, Canada. Ecol. Soc. 17: 20 .

POChETTINO, M. L. \& J. A. HURRELL. 2013. Los cambios ambientales en la región rioplatense (Argentina) y las narrativas de los pobladores locales. Actas X Reunión de Antropología del Mercosur. Córdoba, Argentina (en CD).

RICHERI, M., M. B. CARDOSO \& A. H. LADIO. 2013. Soluciones locales y flexibilidad en el conocimiento ecológico tradicional frente a procesos de cambio ambiental: estudios de caso en Patagonia. Ecol. Austral 23: 184-193.

THE PLANT LIST. 2016. [Permanentemente actualizado, consulta 2016]. The Plant List: A working list of all plant species. Disponible: http://theplantlist.org.

TOLEDO, V. M. 1992. What is ethnoecology? Origins, scope, and implications of a rising discipline. Etnoecológica 1: 5-21.

TOLEDO, V. M. 2002. Ethnoecology: a conceptual framework for the study of indigenous knowledge of nature. En J. R. STEPP, F. S. WYNDHAM \& R. K. ZARGER (eds.), Ethnobiology and Biocultural Diversity, pp. 511-522. University of Georgia Press, Athens.

TORRES ROBLES, S. S. \& M. F. ARTURI. 2009. Variación de la composición y riqueza florística en los talares del Parque Costero del Sur y su relación con el resto de los talares bonaerenses. En J. ATHOR (ed.), Parque Costero del Sur. Naturaleza, conservación y patrimonio cultural, pp 104-121. Fundación de Historia natural Félix de Azara, Buenos Aires.

TORRES ROBLES, S. S \& N. M. TUR. 2006. Los talares de la provincia de Buenos Aires. En A. 
BROWN, U. MARTINEZ ORTIZ, M. ACERBI \& J. CORCUERA (eds.) La situación ambiental argentina 2005. Fundación Vida Silvestre Argentina, Buenos Aires.

TORTORELLI, L. A. 1956. Maderas y Bosques Argentinos. Editorial ACME S.A.C.I., Buenos Aires. 910 pp.

TRINIDADE MEDEIROS, M. F., T. C. da SILVA, R. da SILVA SOUZA \& R. R. VASCONCELOS SILVA. 2014. Oral history in ethnobiology and ethnoecology. En U. P. ALBUQUERQUE, L. V. F. CRUZ DA CUNHA, R. F. P. DE LUCENA \& R. R. NÓBREGA ÁLVES (eds.), Methods and techniques in ethnobiology and ethnoecology, pp. 59-73. Humana Press, New York.
VALDÉS, A. \& D. GARCÍA. 2011. Direct and indirect effects of landscape change on the reproduction of a temperate perennial herb. J. Appl. Ecol. 48: 14221431.

VIEDMA, O., N. MOITY \& J. M. MORENO. 2015. Changes in landscape fire-hazard during the second half of the 20th century: Agriculture abandonment and the changing role of driving factors. Agric. Ecosys. Environ. 207: 126-140.

VITOUSEK, P. M. 1992. Global environmental change: an introduction. Annu. Rev. Ecol. Syst. 23: 1-14.

WANG, J., K. WANG, M. ZHANG \& C. ZHANG. 2015. Impacts of climate change and human activities on vegetation cover in hilly southern China. Ecol. Eng. 81: 451-461.

Original recibido el 14 de abril de 2016; aceptado el 26 de abril de 2016. 
